\title{
An online quality monitoring tool for information acquisition and sharing in manufacturing: requirements and solutions for the steel industry
}

\section{Satu Tamminen*, Eija Ferreira, Henna Tiensuu}

Biomimetics and Intelligent Systems Group, University of Oulu, Finland E-mail: satu.tamminen@oulu.fi

E-mail: eija.ferreira@oulu.fi

E-mail: henna.tiensuu@oulu.fi

*Corresponding author

\section{Heli Helaakoski, Vesa Kyllönen}

VTT Technical Research Centre of Finland Ltd, Oulu, Finland

E-mail: heli.helaakoski@vtt.fi

E-mail: vesa.kyllonen@vtt.fi

\section{Juha Jokisaari}

SSAB Europe Oy, Raahe, Finland

\section{Esa Puukko}

Outokumpu Stainless Oy, Tornio

\section{Juha Röning}

Biomimetics and Intelligent Systems Group, University of Oulu, Finland

E-mail: juha.roning@oulu.fi

\begin{abstract}
The purpose of this study was to develop an innovative online supervisor system to assist the operators of an industrial manufacturing process in discovering new solutions for improving both the products and the manufacturing process itself. In this paper, we discuss the requirements and practical aspects of building such a system and demonstrate its use and functioning with different types of statistical modelling methods applied for quality monitoring in industrial applications. The two case studies presenting the development work were selected from the steel industry. One case study predicting the profile of a stainless steel strip tested the usability of the tool offline, while the other study predicting the risk of roughness of a steel strip had an online test period. User experiences from a test use period were collected with a system usability scale questionnaire.
\end{abstract}

Keywords: data mining; generalized boosted regression; GBM; quality improvement; online monitoring; knowledge representation; product design. 
Reference to this paper should be made as follows: Tamminen, S., Ferreira, E., Tiensuu, H., Helaakoski, H., Kyllönen, V., Jokisaari, J., Puukko, E. and Röning, J. (201X) 'An online quality monitoring tool for information acquisition and sharing in manufacturing: requirements and solutions for the steel industry', International Journal of Industrial and Systems Engineering, Vol. x, No. x, pp.xxx-xxx.

Biographical notes: $\mathrm{S}$. Tamminen received her $\mathrm{PhD}$ in Technology and Embedded systems in 2014 at the University of Oulu. She works as a post doctoral researcher and lecturer in the Biomimetics and Intelligent Systems Group, University of Oulu. Her research interests include industrial data mining, machine learning, artificial intelligence and smart decision support.

E. Ferreira is a Postdoctoral researcher in the Biomimetics and Intelligent Systems Group, University of Oulu, Finland, where she received her $\mathrm{PhD}$ in 2015. Her main research interests include machine learning, data mining, human-computer interaction, wearable and ubiquitous computing, and data quality assessment.

H. Tiensuu is a doctoral student in the Biomimetics and intelligent systems group, University of Oulu, Finland. Her research interests include industrial data mining.

H. Helaakoski works as a Principal scientist in VTT Technical Research Centre of Finland Ltd. She has got her Doctor of Science (Technology, Embedded systems and softwares) in year 2008 from the University of Oulu. Her research interests include artificial intelligence, Internet-of-Things and industrial software systems.

V. Kyllönen is research scientist in VTT Technical Research Centre of Finland Ltd. His main research interests include production planning and monitoring.

J. Jokisaari works as a Senior development engineer at SSAB in Raahe, Finland. $\mathrm{He}$ is an expert in the development of hot rolling process of a steel strip.

E. Puukko works as a Research manager at Outokumpu, Global Stainless Steel producer. His fields of expertise include process areas of FeCr-plant, Melting shop and Hot rolling.

J. Röning is a Professor of Embedded Systems at the University of Oulu. He serves also as a Visiting Professor of Tianjin University of Technology, P. R. China. He is principal investigator of the Biomimetics and Intelligent Systems Group (BISG). His research interests include data mining, machine learning, computer vision, robotics, intelligent signal analysis, and software security.

\section{Introduction}

\subsection{Motivation}

Quality management, delivery reliability and resource efficiency are cornerstones of competitiveness in today's steel industry. Quality, cost and cycle time are the factors that give advantage over one's peers, and among them, quality is particularly critical for getting long-term competitive advantages (He et al., 2009). Recent developments in steel markets, such as new competitors and the need for carbon emission reductions while improving the quality and delivery reliability of products make the operating environment of steel industry more dynamic and complex. On the other hand, recent advances in mathematical modelling 
and IT technologies offer new opportunities for predicting product quality and potential delivery problems in an integrated way and at a larger scale.

Knowledge can be considered the most valuable asset of a manufacturing enterprise when defining itself in the market and competing with others. Manufacturing has benefited from the field of data mining in several areas, including engineering design, manufacturing systems, decision support systems, shop floor control and layout, fault detection, quality improvement maintenance and customer relationship management (Harding et al., 2006). A risk-based approach for quality control planning can help to prevent an excessive scrap production in mass production industries (Bettayeb et al., 2014). The amount of information coming from different sources can easily grow overwhelming, however, which will increase the need for intelligent pre-processing in the systems. Decision support systems, for example, become intelligent when combined with AI tools such as fuzzy logic, casebased reasoning, evolutionary computing, artificial neural networks, and intelligent agents (Phillips-Wren, 2013; Logunova et al., 2014).

Knowledge engineering and data mining have enabled the development of new types of manufacturing systems, and future manufacturing will be able to adapt to demands of agile manufacturing, including a rapid response to changing customer requirements, concurrent design and engineering, lower cost of small volume production, outsourcing of supply, distributed manufacturing, just-in-time delivery, real-time planning and scheduling, increased demands for precision and quality, reduced tolerance for errors, in-process measurements and feedback control (Dumitrache and Caramihai, 2010).

Manufacturing quality improvement requires a strategic approach from the managers. Piatt (2014) presents five steps for eliminating the cost of poor quality. The first one is to adopt a team mind-set. In order to make a lasting and meaningful change in the manufacturing process a team-based approach is needed. When operators from multiple disciplines are engaged in the efforts aiming at quality improvement, a variety of perspectives is obtained. Secondly, quality needs to be defined from the perspective of the customer; the product will not be genuinely better without additional value to the customer. We also need to develop organizational understanding of the cost of quality; the manufacturing staff needs to be trained to understand the cost multipliers in case a defected product reaches a customer. Then, the staff members will be more motivated to find root causes for problems. In the next step, the problems identified need to be solved completely; rather than fixing the symptoms of failure their root causes need to be solved. Finally, the management should employ a strong process discipline. The manufacturing staff will be better motivated to involve themselves into quality improvement if there are tools available to assist in the process. The information should also be easily available and well presented. To conclude, it is important to keep in mind that the process of quality improvement involves team members, managers and operators that all have different objectives and needs for knowledge.

\subsection{Literature review}

When product quality improvement is pursued, Kano and Nakagawa (2008) suggest that a process monitoring system should have at least the following functions: it should be able to predict product quality from operating conditions, to derive better operating conditions that can improve the product quality, and to detect faults or malfunctions for preventing undesirable operation. They used soft sensors for quality prediction, optimization for operating conditions improvement, and multivariate statistical process control (MSPC) for fault detection in a steel industry application. During the last two decades, neural networks 
have been widely used for process modelling and quality prediction as well (Boukezzi, 2017; Bhadesia H., 1999; Tamminen et al., 2013). Also Bayesian decision theory can provide solutions for simple systems, such as monitoring the condition of the manufacturing equipment (Rashidi and Jenab, 2013). Density forecast based predictors are suitable when approximating failure probability for products in manufacturing. Quality models can be extended to density prediction with deviance models that also allow the quality variance to depend on the process settings (Juutilainen et al., 2015).

$\mathrm{Bi}$ et al. (2014) state that every major shifting of the manufacturing paradigm has been supported by the advancement of IT. Internet of Things (IoT) is likely to change the operation and role of many existing industrial systems in manufacturing. Integration of sensors, RFID tags and communication technologies into the production facilities enables cooperation and communication between different physical objects and devices. One of the technical challenges in IoT research is the question of how to integrate IoT with existing IT systems. In addition, when a massive amount of real-time data flow is to be analysed, strong big data analytics skills are also required from the end user (Xu et al., 2014).

In manufacturing, there are several quality parameters that need to be linked with process parameters. There are various widely used methods that are able to monitor the current process variables or product properties factor-wise. Statistical process control (SPC) and automatic process control (APC) can be integrated into process monitoring and adjustment (Akram et al., 2012; Requeijo and Cordeiro, 2013). Control charts can be used to detect changes in the average level of the process or in process variation (Karaoglan and Bayhan, 2014; Pan, 2017). Statistical models give another angle to quality monitoring, as they can be used to predict the future outcome of a process, which in its turn enables planning of the process and the production as a whole. The challenge is the communication between people: how to get the needed information from the process or the product, how to enable information transfer between the work posts or manufacturing facilities, and how to provide information about the malfunction or decreased quality of the products. The information should be presented clearly, offering real solutions to the problems. Warnings should also be provided in case automatic corrective actions are enabled. On the whole, the conversation within a company should be based on the knowledge, and there is a need for tools that can support the information chain.

Poor quality of process data can prevent efficient use of online monitoring, and thus, automatic data preprocessing is needed (Liu et al., 2018). Rodriguez and Servigne (2013) propose a monitoring application where data quality information is provided for the user. Especially in dynamic systems, automatic data cleaning improves the usability of the system.

\subsection{Problem Space}

During a manufacturing process, hundreds or thousands of factors are measured, data is stored into databases, process settings are calculated, and critical variables are monitored using SPC tools. However, interactions and more complex relations between factors often get no continuous attention. There is a need for predictive models to be used from the initial project design and set-up stage through the whole manufacturing process to monitor and optimize the product quality and the flow of the process. New prediction models need to be installed for online use. The predictive models also need to be frequently updated based on the continuously stored process data as the process evolves and changes due, for example, to changes in the operating environment and materials used, and depreciation of 
the manufacturing equipment. There is a need for a platform that is able to operate with different model types and present the results online.

Statistical models effectively process the collected data into information that should be presented differently depending on the end user. Operators need a clear presentation of the current status of the process to be able to make quick decisions and corrections to the course of the process. The implications of the information presentation on their cognitive workload should also be considered to avoid negative effects that too high cognitive load can have on human performance, and ultimately on production outcome (i.e. quality and productivity) (Lindblom and Thorvald, 2014). Engineers in the process development department may need more detailed information about the process, to obtain deeper understanding of the process. Process managers prefer general information and reports from the process and yield. Maintenance personnel on the other hand needs to get early warnings before any malfunctions occur.

In this article, we present a tool for online quality monitoring of manufacturing processes with two application cases in steel industry: predicting the thickness profile of a steel strip and the roughness of a stainless steel surface. Both of these processes have a high variability in product types during the manufacturing, which disables the use of tools developed for mass production for quality improvement. Our tool links together statistical models for quality prediction with the measured process data, and presents the results with easily interpretable visualisations. We performed a system usability study for the first users of the pilot cases. This paper is organized as follows. Section 2 presents the development of the quality monitoring software solution integrating different types of modelling methods for a usable industrial application. The case studies demonstrating the functionalities of the developed tool are presented in Section 3. In Section 4, we report the results and lessons learned from the usability study. Section 5 concludes the quality monitoring application development.

\section{Developing a quality monitoring tool for the manufacturing industry}

\subsection{Domain requirements and requests}

In the beginning of the development process of the quality monitoring tool (QMT), we collected the requirements set by the business, end users and IT environment of the process industry. During the development phase, we arranged a series of workshops with participating companies, development partners and other stakeholders. The main activities for specifying the requirements of the quality monitoring tool were:

- preliminary business requirements,

- company interviews with process development engineers and IT department,

- specifying stakeholders requirements,

- collecting reference materials,

- defining business needs,

- defining the used terminology,

- review by companies in a joint workshop.

In the requirement specification phase, we defined the key user groups that will benefit from the quality system. Technical specifications of the quality tool were defined as follows: 
- stable and reliable applications,

- intuitive, logical and consistent user interface,

- informative information presentation,

- performance,

- maintainability,

- scalability - adding new modules/ features/methods/ algorithms should be easy,

- security,

- authentication,

- recoverability,

- standards and tools - programming languages,

- accessibility - web application, integration to SMC UI also possible.

The QMT prototype is illustrated in Figure 1. The information transfer from the manufacturing process to the end users is presented in four steps: 1) information acquisition 2 ) information storage 3 ) information analysis, and 4) information delivery.

\subsection{Specifications for the tool}

We implemented the models providing quality information for the QMT in R language (The Comprehensive R Archive Network , 2016) and other equations and rules in C++ Mathematical Expression Toolkit Library (ExprTk) (C++ Mathematical Expression Toolkit Library, 2016). $\mathrm{R}$ is a free and open source language for statistical computing integrated into QMT with RServe module which allows other programs to use facilities of R. R scripts can be written standalone and integration into QMT is straightforward. ExprTk is a mathematical expression parsing and evaluation engine directly embedded in the source code of QMT.

We implemented the QMT server side in $\mathrm{C}++$ language. The server side functionality includes data access and integration into models. Online data access for the QMT is accomplished by reading data from a database. The data is pre-processed before use for model calculation. For example, a valid range for the input variables can be set. If these limits are violated and the model result is not reliable, a question mark is shown in the QMT user interface. Depending on the quality monitoring needs on the specific manufacturing line, the system administrators can add new models to QMT.

The web-based QMT user interface provides an overview of the process quality as a starting point. The quality overview highlights the quality status of different process phases with different colours. Typically, red colour indicates process failure or malfunction, yellow flags a warning for process failure and green stands for normal operation. White colour indicates that quality information could not be calculated. The QMT also shows the progress of products through the manufacturing line as a time line labelled with the same colour coding. Figure 2 illustrates the schematic presentation of the server modules of the QMT, as well as snapshots from the user interface. The quality overview hides mathematical models and the numerous process variables from which the quality information is derived. If a process engineer wants to manually find a root cause for a quality deviation, it is possible to go deeper from the quality overview to the actual process variables. QMT also provides the possibility of showing different kinds of views of the quality information for different user groups. 


\subsection{Models for quality and rejection probability prediction}

When selecting a modelling method for quality monitoring, the performance of the model is the most important, but not the only criterion. The method should work with highly non-linear data, but it should also produce information about the relationships between the predicted variable and the explanatory variables. The user is not only interested in the prediction, but also wants to know how the process variables influence the predicted quality. The information provided by the method should be simple and illustrative to guarantee good interpretability of the results. With online systems, the data is rarely flawless. Hence, it is crucial for the operability of the system that the selected methods are able to deal with missing values.

Neural networks have been a popular method for modelling data with complex relationships between variables (Boukezzi, 2017; Bhadesia H., 1999; Tamminen et al., 2013). Lately, ensemble algorithms have risen to challenge them with equal accuracy, faster learning, tendency to reduce bias and variance, but also higher tendency to overfit. Seni and Elder (2010) state that ensemble methods are the most influential development in data mining and machine learning in the past decade. Gradient boosting machines are a family of powerful machine learning techniques that have successfully been applied to a wide range of practical applications (Natekin and Knoll, 2013). Boosted regression trees are capable of handling different types of predictors and accommodating missing data, there is no need for prior transformation of variables, they can fit complex non-linear relationships, and they can automatically handle interactions between predictors (Elith et al., 2008).

We selected the Generalized Boosted Regression Models (GBM) for the quality monitoring task. With this method, relatively poorly performing models, so called 'weak learners', are estimated iteratively and combined together to form a strong learner. The GBM algorithm iteratively fits regression trees to the residual $\hat{\varepsilon}_{i}$, of the current model. The final model is achieved as the sum of the iteratively fitted regression trees. The form of the final model is

$$
y_{i}=\beta_{0}+\sum_{k=1}^{H} \lambda T_{k}\left(x_{i}\right)+\hat{\varepsilon}_{i},
$$

where each $T_{k}\left(x_{i}\right)$ is a regression tree predictor with $K$ terminal nodes. The predicted value at each terminal node is constant. The learning process is controlled by the shrinkage parameter $\lambda$, the depth of a single tree $K$ and the number of trees $H$. These meta-parameters can be optimized to maximize the prediction accuracy on the validation data. Details on the iterative process in which the single trees $T_{k}\left(x_{i}\right)$ are fitted are given by Friedman (2002).

Probabilistic forecasting methods that predict a risk of failure in meeting the product specifications provide visually powerful information for the QMT. They can be used to predict the disqualification risk $R_{i}=1-P\left(Y_{i}^{\text {min }} \leq y_{i} \mid x_{i}\right)$ in a quality control test, for example. We used this approach for predicting the risk of a hazardous shape of the product. The predicted disqualification risk can be calculated from the predicted conditional cumulative distribution function $F_{y_{i} \mid x_{i}}=F\left(s, x_{i}\right)=P\left(y_{i}<s \mid x_{i}\right)$, where $s$ is a predefined rejection or exceedance limit.

If the distribution of the standardized residuals is assumed to be normal for the mean model, the probability of rejection can be calculated with

$$
P_{i}=\Phi\left(\frac{L-\hat{\mu}_{i}}{\hat{\sigma}}\right)
$$


where $\Phi$ is the cumulative normal distribution function, $\hat{\mu}_{i}$ is the quality property estimate and $\hat{\sigma}$ is the standard deviation. The rejection probability risk level that will lead to a change in product design should be low enough to recognize the unsuccessful products and high enough not to trigger too many false alarms. The method can be expanded to cases with non-Gaussian distributions as well (Juutilainen et al., 2012).

In industrial applications, the assumption of constant variance $\hat{\sigma}^{2}$ for the whole range of production is unrealistic, and thus, not only the mean but also the variance of the predicted property can be dependent on the explanatory variables. Variance modelling has been used, for example, in quality improvement experiments designed to map the process settings that minimize variance under given conditions. Variance modelling is also naturally suited for tolerance design, because it helps to find the sources of variance in the process (Engel, 1992; Tamminen et al., 2010). The purpose of variance function estimation is to model the structure of the variances as a function of predictors (Carroll and Ruppert, 1988). If the response variable of a model has a distribution belonging to the exponential family, Generalized Additive Models (GAM) can be used for the modelling. These nonparametric extensions of Generalized Linear Models (GLM) operate with a sum of smooth functions $\sum s_{j} X_{j}$, where $s(\cdot)$ 's are unspecified functions that are estimated using a scatter plot smoother (Hastie and Tibshirani, 1986; Hastie et al., 2001). We used variance models to improve the performance of the risk probability prediction models.

\section{Quality monitoring in manufacturing}

We selected two different case studies from the steel industry to demonstrate the properties, functionalities, and the online use of our quality monitoring tool. However, it should be noted that the developed QMT is not application dependent, but can be deployed in other manufacturing areas as well.

\subsection{Case studies}

The industrial plant of our first case study produces high quality steel products with a short delivery time. As a result, the variability of their product grades during the manufacturing is large, and process optimization methods designed for mass manufacturing cannot be applied. The target profile of a steel strip is a quality property that is set in collaboration between the product development and the customer, depending on the further processing steps planned for the product. Especially for cold rolling, a negative profile can be very harmful. The target profile is typically set between $0.03-0.08 \mathrm{~mm}$, and without the advantage of mass production, the target can vary from product to product during rolling. Thus, it is more difficult to hit the target without a strip to strip profile adaptation. With prediction models, we can design products that more likely fulfil the requirements and find root causes for failures. In our QMT, the user can select between the profile and the deviation from the target profile models, depending on the needs. For quality monitoring purposes the profile of a steel strip as well as the deviation from the target profile was modelled in three process steps: after furnace, after roughing mill and after finishing mill. Accuracy of the profile modelling improves at the end of the process where the most influential variables are available, but with these models, it is also possible to give an early warning based on the preceding process steps. Thus, the models provide the user a better insight of the effect of process parameters and they can also be used to test the user's prior assumptions or understanding of the process. 
Our second use case relates to the surface roughness of a stainless steel strip, which can only be detected after the strip has been polished even though the defect might have come into being during the hot rolling stage already. After hot rolling, the product goes through cold rolling, different surface treatments and finally polishing, and these following process steps may also worsen the defect. In this use case, the plants for hot and cold rolling are located in different countries, and it is therefore crucial to identify the products that will not meet the quality requirements due to defects caused by hot rolling already before the shipping. With the QMT, the user will be able to reallocate the high risk coils for other end users with lower surface quality requirements. It is equally important to detect the process settings that increase the risk of surface roughness and to re-adjust the process if systematic quality failures occur.

\subsection{GUI functionality for the quality monitoring tool}

The most important feature of the QMT is its ability to simultaneously visualise results from a large number of prediction models. Thus, the information about the predictions has to be presented in a simple way. Each single product is visualised with a bar with height and colour corresponding to its individual quality prediction. We selected the welltrodden traffic light colours green, yellow and red for characterising the predicted quality. Additionally, the height of the bar corresponds to the magnitude of the predicted value in relation to the colour class. The user can set the time span preferred for monitoring, which in its turn enables observation of the production in a meaningful way depending on their interests. The number of displayed models can be selected as well.

The QMT user interface for steel strip profile monitoring is presented in Figure 3. The online view of the manufacturing process augmented with quality information can be seen on the bottom of the window. The user can easily zoom in to the products for more detail. This is especially helpful if the selected time period is long or the number of selected models is large. Above the process view, there is a summary of the corresponding predictions of the models at different process steps. The user can select basic time-line plots for each process variable during the selected period, or fetch more information of each product by clicking on the corresponding bar.

\subsection{The inspection of the quality property with prediction models}

We selected the GBM models for quality prediction because they provide detailed information about the relative influence of the individual variables on the predicted quality of the product. As the total number of process variables in a model is generally high, this listing helps to find the variables that could be adjusted for quality improvement. The list of the most influential variables in the surface roughness use case is presented in Figure 4.

The effect of each variable in the model on the dependent variable can be visualized with partial dependence plots (PDP) (Friedman, 2001). Figure 5 presents the effect of one chemical component, niobium (left), and the set target profile (right) on profile deviation. An increase in the amount of niobium increases the risk of positive profile deviation, which may relate to the strength of the steel. The profile target on the other hand seems to have a negative correlation with the profile deviation. With the QMT, the user can learn about the process and how the process variables affect the quality of the product.

The interactions between variables are equally interesting. With the QMT's visualization functionalities, the user can locate parameter ranges in which the risk of failure in the 
production is the lowest. Figure 6 illustrates the co-effect of the two aforementioned variables important for the strip profile. The figure suggests that the risk of positive profile deviation caused by niobium could be cancelled out by setting a higher target profile for the product.

PDPs are a reliable and useful method for examining a selected subset of variables in case their dependence on the remaining predictive variables is not too strong. In industrial applications, this assumption is easily violated and instead, individual conditional expectation (ICE) plots can be used. They display the estimated functional relationship separately for each observation instead of averaging it over the variables to one curve. The thick line in the middle corresponds to PDP (compare with Figure 5). The partial derivative ICE plots (d-ICE) enable finding regions of interactions (ROI) in the observed variables (Goldstein et al., 2015). In Figure 7, the effect of the variable 'specific force on 6th pass' on profile deviation can be examined for each observation in an ICE plot (left). It can be seen in a d-ICE plot (right) that this variable has the strongest interactions with other variables when its values fall between 7 and 8 .

The ICE plot for one product group in the roughness use case shows an interesting behaviour pattern as the curves are clustered to two groups in Figure 8. Colour coding with another variable could help to find out the cause for this, but especially in industrial applications, the interaction of two or more predictive variables is a more probable explanation. From the user's point of view, this plot brings up the question of the cause and motivates further investigation with other methods.

Risk probability predictors are effective tools in monitoring systems. The accuracy of a prediction can be improved with a distribution shape model, because not only the mean but also the deviation of the modelled quality property can be dependent on the predicting variables. In cold rolling of a steel strip, a negative profile is especially harmful, and we therefore implemented an alarm for the risk of negative profile into the monitoring tool. The predicted risk can be presented among other models in GUI (Figure 3).

Parallel coordinates (Inselberg, 1998) are a visual data mining method that transforms a search for relationships in a multivariate data set into a 2D pattern recognition problem. Thus, it enables a simultaneous visual inspection of several factors in a quality model. In our QMT, the user can select some interesting factors for visualization and thus effortlessly check how their hypotheses would realize with a larger set of historical data that enables a comprehensive analysis of the process with a higher amount of similar products. Figure 9 presents a parallel coordinates plot for one product from the roughness use case. Each line in the plot represents one observation. The leftmost column is the predicted roughness, and the following columns are the selected factors. Observations with high predicted roughness have been plotted in red. As we can see, the predicted roughness associates strongly with a high reduction on the 10th pass, short heating time for slabs and a large roll diameter on the 5th pass. The cumulative effect of these factors might be the reason for the gap visible in Figure 8.

\subsection{Online inspection of a single observation}

With our QMT, the user can examine the properties of each product by clicking on the corresponding bar in the overview of the manufacturing line. In addition to the basic background information of the product, the user can compare its details to other products from the same product type with higher predicted quality using parallel coordinates. This feature is especially useful when the models predict an alarmingly low quality. The user 
can then hand pick the variables to be inspected, or choose the most important variables from the GBM model. In Figure 10, two products from the target profile use case with a low predicted quality are compared with similar products with high predicted quality. In the upper case, the plot reveals that the process variables 2-4 should be considered as strong candidates for causing a positive deviation from the target profile. In the lower case, there are no clear candidates in the process variables that could have caused a negative deviation from the target profile.

In online use, it is not uncommon that some of the process data is occasionally not stored into the database, and the tool has to proceed with incomplete information. In that case, it is important to notify the user that the data has missing values in some of the factors of the quality model. These predictions are indicated with a question mark. Thus, the user is aware of the need for checking whether the incomplete factor has a strong impact in the model or not, and thus, it is possible to evaluate how reliable the prediction is actually. Factors with higher relative influence being missing from the model are more likely to distort the result than factors with low influence. It is also possible, that some models cannot be used for all of the product types. For example, the selected quality property may not be relevant for a certain product group. Then, only white colour is displayed for that model. Figure 11 presents the QMT user interface in actual online use for surface defect monitoring with a period of missing values in one of factors.

\section{Usability evaluation with the System Usability Scale}

In tool development, it is important to get feedback from the users. Furthermore, it would be valuable if the results would be comparable with the ones obtainable with alternative tools. The System Usability Scale (SUS) is a standardized questionnaire widely used to evaluate the usability of various kinds of applications. It gives a global view of subjective assessments of usability, and it is generally performed after the respondent has had an opportunity to use the system, but before any debriefing or discussion takes place (Brooke, 1996).

The SUS questionnaire consists of ten statements that characterise either positive or negative aspects of the system. The respondent is asked to select their immediate response to each statement on a scale from 1 to 5 from strongly disagree to strongly agree. If they cannot respond to a particular statement, they are advised to choose the centre value. The statements are as follows:

1. I think that I would like to use this system frequently.

2. I found the system unnecessarily complex.

3. I thought the system was easy to use.

4. I think that I would need the support of a technical person to be able to use this system.

5. I found the various functions in this system were well integrated.

6. I thought there was too much inconsistency in this system.

7. I would imagine that most people would learn to use this system very quickly.

8. I found the system very cumbersome to use.

9. I felt very confident using the system.

10. I needed to learn a lot of things before I could get going with this system.

We tested an early-phase prototype of the QMT with five process engineers, who filled in the SUS questionnaire after a short test use period. On average, the users had only spent 
a couple of hours testing the tool. Our goal was not to get final user experiences about the QMT, but to get valuable feedback for the design and development of the tool.

On a scale of $0-100$, the average of the results was 58 , while the highest score was 90 , and the lowest score was 37.5. One of the users was very satisfied with the tool, while another had highly negative feelings about it. There were quite many responses with the middle option selected indicating that the respondent either did not have an opinion about the statement or did not have enough experience with the system due to the shortness of the test period. However, the results clearly indicated where we had already succeeded and where we needed to improve.

The first question showed a duality in the reception of the tool. Four of the test users strongly or slightly agreed and one of them strongly disagreed that they would like to use the system frequently. In the second question, the test users were more unanimous: three of them felt neutral and two of them slightly disagreed about the system being unnecessarily complex. The third question received a neutral score from two users, while one slightly agreed and one slightly disagreed about the system being easy to use. The fourth question showed that some users would need some technical support to be able to use the tool, while others thought they would manage by themselves. In the fifth question, two of the users felt that we could improve the integration of the functions in the system, while in the sixth question, none of the users found too many inconsistencies in the system. The question seven divided the opinions again with all but one of the test users slightly or strongly agreeing that they could imagine most people learning to use the system very quickly, but one strongly disagreeing with the statement. Only one slightly agreed that the system was cumbersome to use in question eight. In the last two questions, two of the users did not feel confident using the system, and only one slightly agreed that there were are lot of things to learn before they could start using the system.

Even though the SUS is not meant to be used as a diagnostics tool, these results nevertheless gave us valuable information on how to improve the usability of the system. The purpose of the QMT is to bring information about the manufacturing process from process control tools, statistical models and the process data base available to the users. As a user might not have much prior knowledge or experience with the models used, it is natural that it might take some time to familiarise oneself with the system and to get the most benefit from the information offered. The general process overview of the QMT is relatively easy to interpret and it allows for diverse exploration of the product and quality information offered by the models. However, if needed, the tool may be developed into more advisory direction in the future.

Collaboration between experts of both data mining and the specific manufacturing area is needed for a successful industrial application (Wang, 2007). Usually, data mining experts do not have deeper knowledge of the application area, and similarly, the expert of the application area may not have the skills for performing powerful data analysis. As a result of the successful collaboration, we will be able to deliver a quality monitoring tool that will meet the needs set by the partners during the requirement specification phase. Also, the operators will be motivated to use the tool frequently, as they have been involved in the very development process. 
An online quality monitoring tool for information acquisition and sharing

\section{Conclusions and future work}

We developed an online quality monitoring tool for information acquisition and sharing in manufacturing. The tool is aimed at industrial lines that have a high variability in product types being manufactured, which prevents the use of conventional tools used in mass production. Our tool responds to the demands for automated process data analysis and thus decreases time-consuming manual work. An important property of the tool is that it warns about an increased risk for low quality early in the manufacturing process, when there is still time to avoid production losses by changing the process settings. It also facilitates troubleshooting while trying to find root causes for quality deterioration. We built several statistical models for quality prediction and process analysis and implemented them on the tool. The models are accessible with a web based user interface. We applied the tool in two different steel making processes and tested it both offline and online with engineers working at process development. Their experiences have been a valuable resource for further development of the tool.

We chose an easy-to-interpret presentation for the modelling results with a three colour coding. Thus, it is possible to get an overview of the process status with several quality predictions at once. Our tool also enables localisation of problems in production. When the user notices a deviant product or a production period, it is easy to fetch more information about the product by selecting suitable actions from the menu. Feedback collected from the users for the process overview was positive. There is still a need for an improvement with the detailed analysis of the process, of course. We have already included several visualization methods for the models into the QMT, but for example, the automated root cause analysis needs more work in the future. During offline and online test periods, the users commended positively the general view of the process as it managed to present the overview of several models at once, and it enabled the location of problems in production. The test periods are still ongoing.

During a manufacturing process, the collected data may be stored into different databases and the data format may change; missing values may be coded differently, and even the character for a decimal point may vary. In our case, it took a long time to build the connections to the actual process databases, and we noticed that all the data was not continuously available. Thus, we had to develop ways to indicate that the reliability of the predictions may sometimes be decreased due to incomplete data. Automatic checking for data quality is also important. For example, the measurements may be out of the range of the data set used for training the models for several reasons; the product may be rare and made with unusual process settings, or erroneous measurements may have been stored. In either case, the user should be aware of the situation to be able to act accordingly.

Our next step will be to engage different user groups to the development process. For example, operators monitor several screens channelling information from the production line, and thus, their capacity to pay attention to additional information is limited. Especially, when an alarm for low quality is triggered, the system should provide assistance on what they could do to improve the situation. In addition, the search for process variables affecting the quality could be narrowed down to the ones the operators can actually control during the manufacturing. We will further develop the proposed parallel coordinates approach for smart decision support.

In the current version of the QMT, a selected production period or a single product can be compared with a sample fetched from a historical data set that has a large presence of different product types. Later, we will improve the dynamicity of the tool by allowing 
fetching of up to date comparison data from the online data base. This way, the user will be able to compare the current process status to the past month, for example. Or, the tool will be able to demonstrate how the previous fifty similar products were manufactured compared to the current product giving a quality alarm. As a result, it will be faster to find process settings potentially causing quality issues in a constantly changing environment.

The development in industrial Internet constantly provides new solutions for advanced manufacturing. In the future, intelligent decision-making will become established in industries, and models producing process knowledge will become even more valuable. A shift from manufacturing towards services is under way, also in the steel industry. In the future, quality information of the products will be a selling point, and thus, it should be accessible during the manufacturing process.

\section{References}

Akram, M.A., Saif, A-W.A. and Rahim, M.A (2012) 'Quality monitoring and process adjustment by integrating SPC and APC: a review', International Journal of Industrial and Systems Engineering, Vol. 11, No. 4, pp. 375-405.

Bettayeb, B., Bassetto, S.J. and Sahnoun, M. (2014) 'Quality control planning to prevent excessive scrap production', Journal of Manufacturing Systems, Vol. 33, pp. 400-411.

Bhadesia H. (1999) 'Neural Networks in materials science', ISIJ International, Vol. 39, No. $10,966-979$.

Bi, Z., Xu, L. and Wang, C. (2014) 'Internet of things for enterprise systems of modern manufacturing', IEEE Transactions on Industrial Informatics, Vol. 10, No. 2, pp. 15371546.

Boukezzi, F., Noureddine, R., Benamar, A. and Noureddine, F. (2017) 'Modelling, prediction and analysis of surface roughness in turning process with carbide tool when cutting steel C38 using artificial neural network', International Journal of Industrial and Systems Engineering, Vol. 26, No. 4, pp. 567-583.

Brooke, J. (1996) 'SUS: A "quick and dirty" usability scale', in Jordan, P.W. et al (Eds.), Usability Evaluation in Industry, Taylor and Francis, London, pp. 189-194.

C++ Mathematical Expression Toolkit Library. [online] http://www.partow.net/programming/exprtk/ (accessed 17 February 2017).

Carrol, R. and Ruppert, D. (1988) Transformation and Weighting in Regression, Chapman and Hall, USA.

Dumitrache, I. and Caramihai, S.I. (2010) 'The intelligent manufacturing paradigm in knowledge society', in Virtanen P. and Helander N. (Eds.), Knowledge Management, InTech, pp. 36-56.

Elith, J., Leathwick, J.R. and Hastie, T. (2008) 'A working guide to boosted regression trees', Journal of Animal Ecology, No. 77, pp. 802-813.

Engel, J. (1992) 'Modelling variation in industrial experiments', Applied Statistics, Vol. 41, No. 3, pp. 579-593. 
Friedman, J. (2001) 'Greedy function approximation: A gradient boosting machine', The Annals of Statistics, Vol. 29, No. 5, pp. 1189-1232.

Friedman, J. (2001) 'Stochastic gradient boosting', Computational Statistics and Data Analysis, No. 19, pp. 367-378.

Goldstein, A., Kapelner, A., Bleich, J. and Pitkin, E. (2015) 'Peeking inside the black box: visualizing statistical learning with plots of individual conditional expectation', Journal of Computational and Graphical Statistics, vol. 24, No. 1, pp. 44-65.

Harding, J.A., Shahbaz, M., and Srinivas, S. and Kusiak, A. (2006) 'Data mining in manufacturing: a review', Journal of Manufacturing Science and Engineering, No. 128, pp. 969-976.

Hastie, T. and Tibshirani, R. (1988) 'Generalized Additive Models', Statistical Science, Vol. 1, No. 3, pp. 297-318.

Hastie, T., Tibshirani, R. and Friedman, J. (1988) The Elements of Statistical Learning: Data Mining, Inference, and Prediction, Springer, New York.

He, S., Wang, G.A. and Li, L. (2009) 'Quality improvement using data mining in manufacturing processes', in Ponce J. and Karahoca A. (Eds.), Data Mining and Knowledge Discovery in Real Life Applications, I-Tech, Austria, pp. 357-372.

Inselberg, A. (1998) 'Visual data mining with parallel coordinates', Computational Statistics, Vol. 13, No. 1, pp. 47-63.

Juutilainen, I., Tamminen, S. and Röning, J. (2012) 'A tutorial to developing statistical models for predicting disqualification probability', in Davim, J.P. (Ed.), Computational Methods for Optimizing Manufacturing Technology, Models and Techniques, IGI Global, USA, pp. 368-399.

Juutilainen, I., Tamminen, S. and Röning, J. (2015) 'Density forecast based failing probability predictors in manufacturing' European Journal of Industrial Engineering, Vol. 9, No. 4, pp. 432-449.

Kano, M. and Nakagawa, Y. (2008) 'Data-based process monitoring, process control, and quality improvement: recent developments and applications in steel industry', Computers \& Chemical Engineering, Vol. 32, No. 1-2, pp. 12-24.

Karaoglan, A.D. and Bayhan, G.M. (2014) 'A regression control chart for autocorrelated processes' International Journal of Industrial and Systems Engineering, Vol. 16, No. 2, pp. 238-256.

Lindblom, J. and Thorvald, P. (2014), 'Towards a framework for reducing cognitive load in manufacturing personnel', Proc. of International Conference on Applied Human Factors and Ergonomics (AHFE 2014), pp. 6267-6278.

Liu, X., Tamminen, S., Siirtola, P., Röning, J., Riekki, J., Kiljander, J. and Soininen, J.P. (2018) 'Enhancing Veracity of IoT Generated Big Data in Decision Making' Proc. IEEE International Conference on Pervasive COmputing and Communications (PerCom 2018). 
Logunova, O.S., Matsko, I.I., Posohov I.A. and Luk'ynov S.I. (2014) 'Automatic system for intelligent support of continuous cast billet production control processes', International Journal of Advanced Manufacturing Technology, Vol. 74, pp. 1407-1418.

Natekin, A. and Knoll, A. (2013) 'Gradient boosting machines, a tutorial', Frontiers in Neurorobotics, Vol. 7, Article 21, pp. 1-21.

Pan, X. (2017) 'Horizontal cumulative variance chart: a quality control scheme monitoring shifts in process variation' International Journal of Industrial and Systems Engineering, Vol. 35, No. 2, pp. 265-277.

Phillips-Wren, G. (2013) 'Intelligent Decision Support Systems', in M. Doumpos and E. Grigoroudis (Ed.), Multicriteria Decision Aid and Artificial Intelligence, John Wiley \& Sons, Ltd, Chichester, UK.

Piatt, J. (2014) 'Five steps to improved manufacturing quality', Industry Week, Vol. 263, No. 10, pp. 22.

Rashidi, K. and Jenab, K. (2013) 'Intelligence-based condition monitoring model' International Journal of Industrial and Systems Engineering, Vol. 13, No. 2, pp. 250-261.

Requeijo, J.G. and Cordeiro, J. (2013) 'Implementation of the statistical process control with autocorrelated data in an automotive manufacturer' International Journal of Industrial and Systems Engineering, Vol. 13, No. 3, pp. 325-344.

Rodrigues, C.C.G and Servigne, S. (2013) 'Managing Sensor Data Uncertainty: a data quality approach' International Journal of Agricultural and Environmental Information Systems, Vol. 4, No. 1, pp. 35-54.

Seni, G. and Elder, J.F. (2010), 'Ensemble methods in data mining: improving accuracy through combining predictions', in Grossman R. (ed.) Synthesis Lectures on Data Mining and Knowledge Discovery, Morgan \& Claypool, USA, pp. 1-108.

Tamminen, S., Juutilainen, I. and Röning, J. (2010) 'Modelling of Charpy V test rejection probability', Ironmaking and Steelmaking, Vol. 37, No. 1, pp. 35-40.

Tamminen, S., Juutilainen, I. and Röning, J. (2013) 'Exceedance probability estimation for quality test consisting of multiple measurements', Expert Systems with Applications, Vol. 40 , pp. 4577-4584.

The Comprehensive R Archive Network. [online] http://cran.r-project.org/ (Accessed 17 February, 2017).

Wang, K. (2007) 'Applying data mining to manufacturing: the nature and implications', Journal of Intelligent Manufacturing, Vol. 18, No. 4, pp. 487-495.

Xu, L.D., He, W., Li, S. (2014) 'Internet of things in industries: a survey', IEEE Transactions on Industrial Informatics, Vol. 10, No. 4, pp. 2233-2243. 


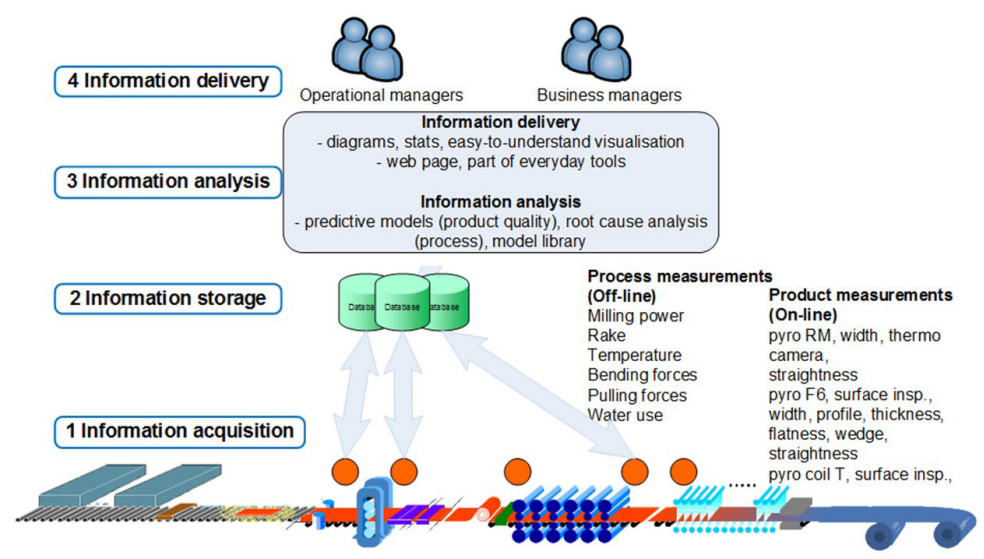

Figure 1 QMT prototype.

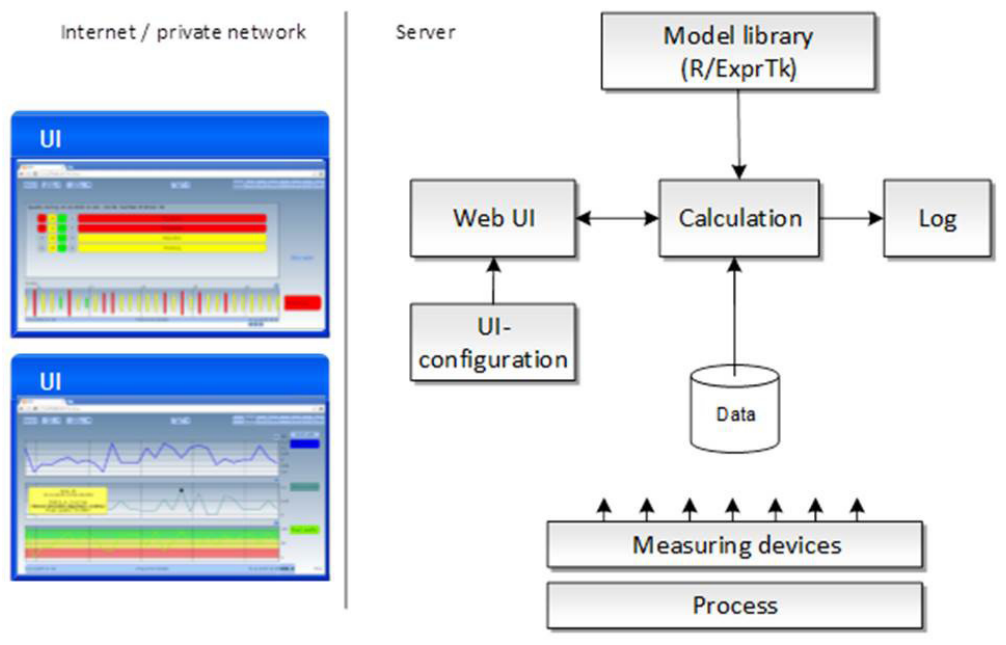

Figure 2 Achitecture of the QMT. 


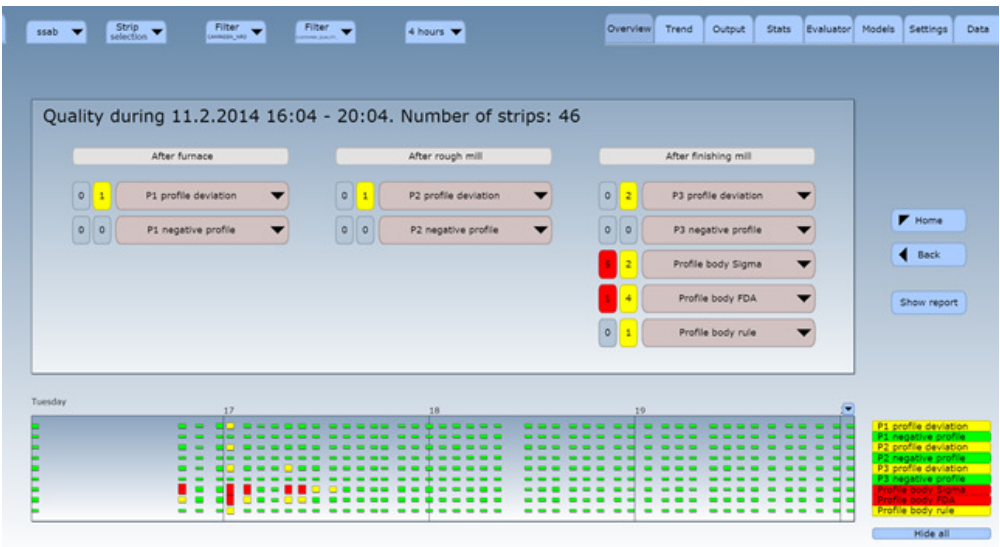

Figure 3 The user interface of the QMT for strip profile monitoring.

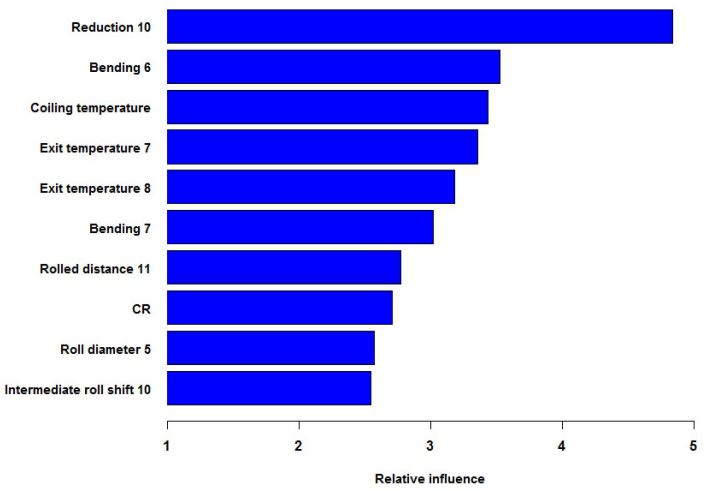

Figure 4 The relative influence of the ten most important features in the roughness model.
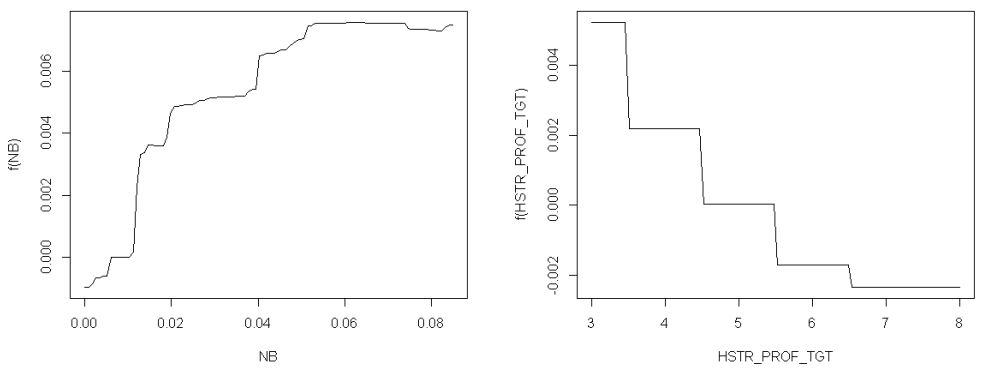

Figure 5 Visualization of the effect of niobium (left) and target profile (right) on profile deviation. 


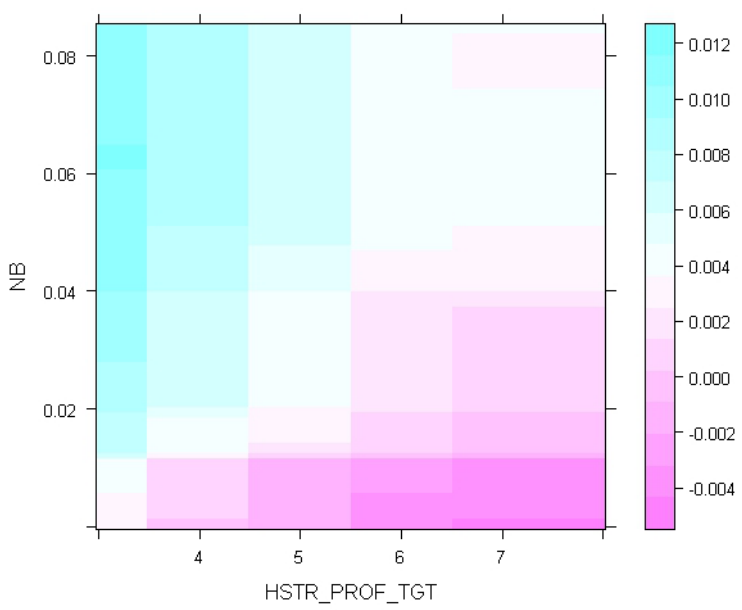

Figure 6 A visualization of the co-effect of niobium and target profile on profile deviation.
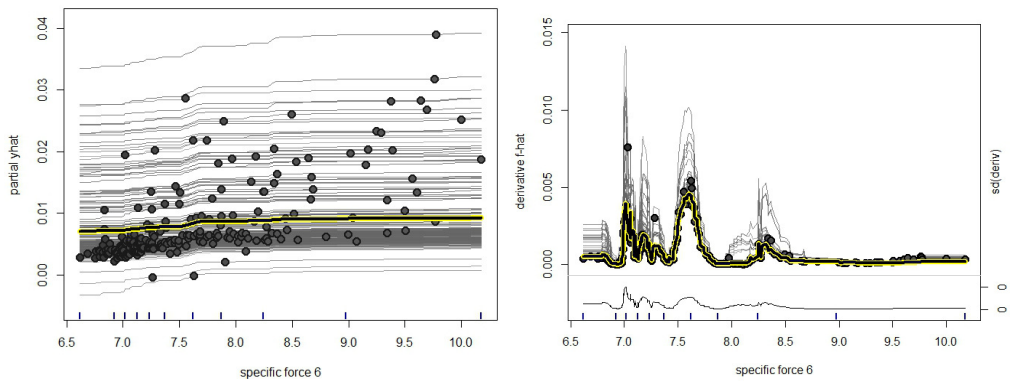

Figure 7 ICE and d-ICE plots for one product group in the strip profile use case.

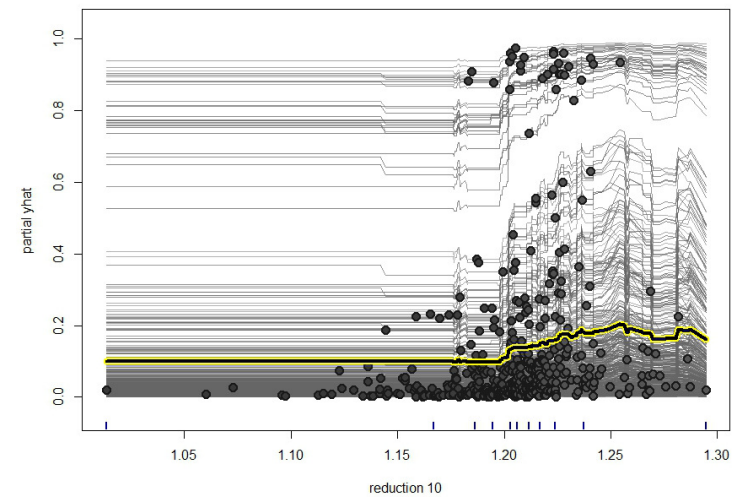

Figure 8 An ICE plot for one product group in the roughness use case. 


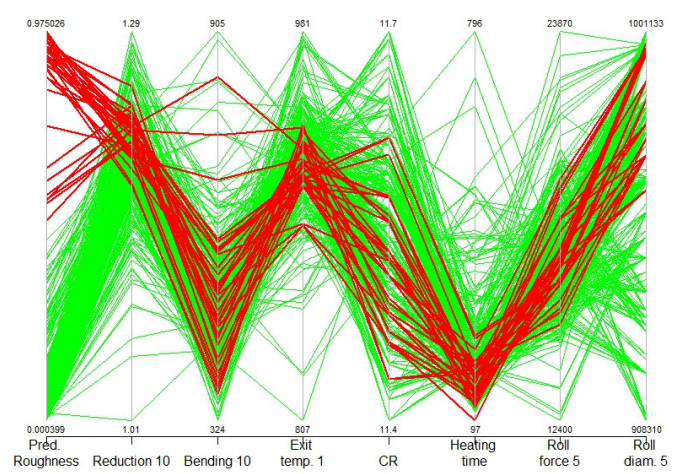

Figure 9 A parallel coordinates plot for a product with a low predicted risk of roughness (observations with high predicted roughness are plotted in red).
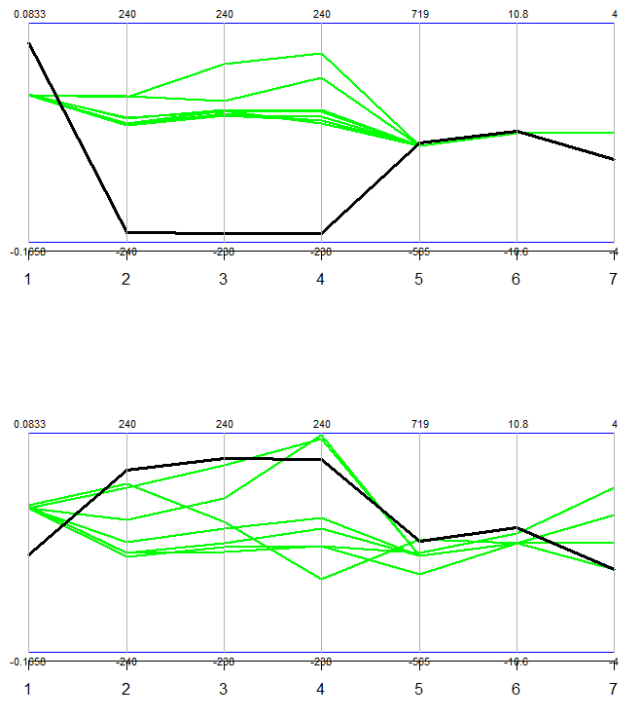

Figure 10 The observed product (black) can be compared with the similar good products (green) by using parallel coordinates. 


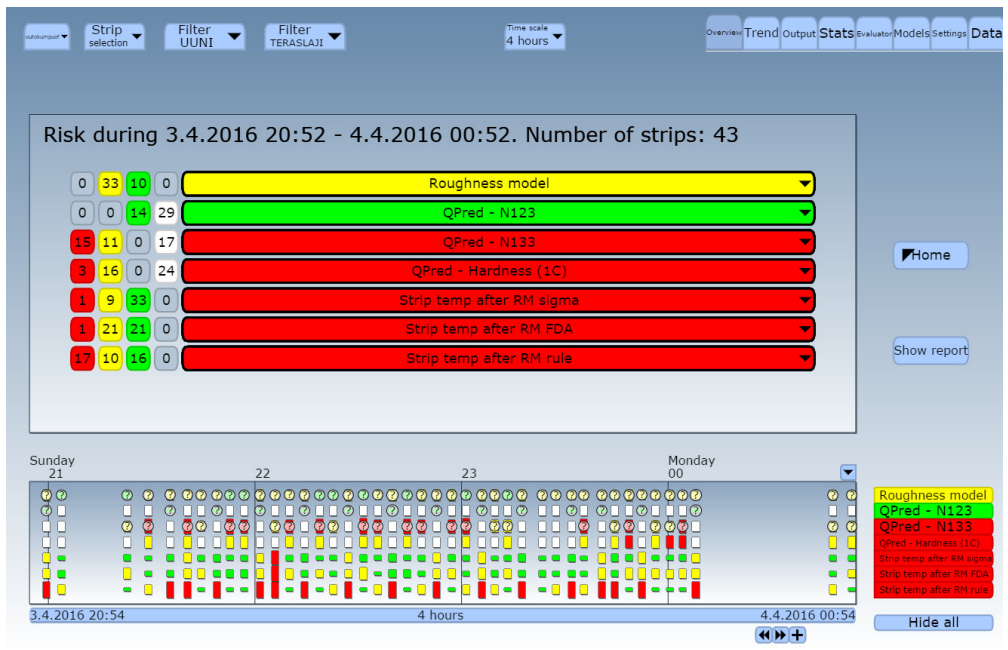

Figure 11 The online view of the QMT for surface defect monitoring. 